\title{
Fischzüge der Liebe: Liebeskommunikation in deutschen und chinesischen SMS-Sequenzen
}

\author{
Wolfgang Imo (Duisburg/Essen)
}

\begin{abstract}
One of the biggest problems with love talk is that the lovers want to make sure of their true and sincere love but that at the same time the assertion of truth and sincerity may also be interpreted as mere routine and the utterance of clichés. On the basis of a qualitative analysis of German and Chinese love communication by SMS it will be shown from a cross-linguistic and cross-cultural perspective how interactants cope with this dilemma. The data show that paradoxically - one solution is to tease each other and produce intentional misunderstandings that yield low puns (a strategy found in the German data) or to enact the end of the love relationship (in the Chinese data).
\end{abstract}

\section{$1 \quad$ Einleitung}

Die SMS-Kommunikation ist als dyadische und primär private Kommunikationsform prädestiniert dazu, neben dem Telefonat - sei es mobil oder per Festnetzanschluss -, dem Brief bzw. der E-Mail und natürlich der nicht medial vermittelten Face-to-FaceKommunikation eine zentrale Rolle für die Liebeskommunikation zu spielen. Mit Hilfe des immer bereiten und schnell einsatzfähigen Mediums Mobiltelefon und der damit verfügbaren Kommunikationsform SMS lassen sich neu entstehende Liebesbeziehungen festigen, bestehende Beziehungen aufrecht erhalten und schließlich im Extremfall auch scheiternde Beziehungen abrupt beenden.

In dem vorliegenden Beitrag sollen sprach- und kulturvergleichend einige ausgewählte Aspekte der Formen und Funktionen der Liebeskommunikation in bereits bestehenden Beziehungen diskutiert werden. Dafür wurden exemplarisch deutsche und chinesische SMSSequenzen von Paaren in festen Beziehungen analysiert (mehr zu den Daten in Abschnitt 3). Liebeskommunikation von solchen Paaren dient typischerweise der Aufrechterhaltung der bestehenden Beziehung. Ein Faktor, der grundlegend für Liebes-SMS ist, besteht dabei in der von Luhmann (1994: 199) konstatierten Gefahr intimer Beziehungen, die in ihrer Instabilität besteht (ausführlich hierzu auch in Bezug auf die gesprochene Sprache Auer 1988). Die emotional bedingte Instabilität von Liebesbeziehungen findet ihren Niederschlag in lexikalischen, syntaktischen und sequenziellen Strukturen, die dem Ausdruck von Liebe und der Aushandlung der gemeinsamen Definition der Liebesbeziehung dienen. Missverständnisse und ihre Behebung, Trauer und Wut über vermeintliches Fehlverhalten des Partners/der Partnerin, aber auch auf der anderen Seite Freude, Vertrautheit und Sehnsucht spielen in der Liebeskommunikation in besonderem Maße eine Rolle. Um die

\footnotetext{
* Dieser Beitrag ist im Rahmen des von der DFG und dem China Scholarship Council geförderten "projektbezogenen Personenaustauschs" der Universitäten Münster und Xi' an mit dem Titel "Kommunikation in den Neuen Medien: Eine kontrastive Untersuchung von deutschen und chinesischen SMS-Botschaften" entstanden. Ich danke Susanne Günthner, Katharina König, den TeilnehmerInnen der Tagung "Sprache in den Medien" an der Xi'an International Studies University (VR China) sowie den beiden anonymen GutachterInnen von Linguistik online für ihre hilfreichen Kommentare.
} 
"unwahrscheinliche Anforderung" von Liebeskommunikation zu bewältigen, muss auch stets mit einem Zusammenbruch der Kommunikation gerechnet werden: "Wie immer bei unwahrscheinlichen Anforderungen ist ein gelegentlicher, wenn nicht häufiger Zusammenbruch der Kommunikation wahrscheinlich." (Luhmann 1994: 45)

In dem vorliegenden Beitrag sollen einige der Strategien analysiert werden, mit denen chinesische und deutsche SMS-SchreiberInnen ihre Liebesbeziehung aufrechterhalten. Besonders auffällig war dabei ein Muster in den chinesischen SMS, das in einer inszenierten, ritualisierten Aufkündigung der Beziehung bestand. In den deutschen SMS-Daten ließ sich ein vergleichbares Ritual dagegen nicht finden, allerdings gibt es dort Rituale vor allem des "Frotzelns" und "Kalauerns", die einen ähnlichen Zweck verfolgen.

\section{SMS: Medium, Kommunikationsform und Gattung}

In der Forschung zu computervermittelter Kommunikation muss unterschieden werden zwischen

i. den Faktoren, die in durch die verwendete Technik bestimmt sind und die die Speicherung, Übertragung, Möglichkeiten der Weiterverarbeitung etc. der Nachrichten betreffen,

ii. den Faktoren, die auf einer konkret bestimmbaren Ebene die Optionen und Grenzen setzen, wie genau kommuniziert werden kann (z. B. ob nur Text oder Text und Bild kombiniert werden können oder ob die Nachricht unmittelbar oder mit Verzögerung angezeigt wird etc.) und

iii. den Faktoren, die typisch für die sprachliche, inhaltliche, funktionale und situationale Ausprägung bestimmter Kommunikationsstrukturen sind. In Anlehnung an Dürscheid (2005) sollen für diese drei Faktorenbündel die Begriffe des Mediums, der Kommunikationsform und der kommunikativen Gattung verwendet werden.

Bei der Definition des Begriffs Medium stützt sich Dürscheid auf Holly (1997: 70), der Medien definiert als "konkrete materielle Hilfsmittel, mit denen Zeichen verstärkt, hergestellt, gespeichert und/oder übertragen werden können."1 Dieses "technologische Medienkonzept" (Dürscheid 2005: 2) zielt darauf ab, den Medienbegriff auf von Menschen hergestellte Hilfsmittel zu beschränken. Schall wird somit nicht als Medium angesehen, wohl aber ein Lautsprechersystem. Für die in dieser Untersuchung behandelte SMS-Kommunikation können die relevanten Medien als die Mobiltelefone (inklusive ihrer Mikroprozessoreinheiten, ihrer Speicher, ihrer Software und ihrer Netze) bestimmt werden. Das Medium hat dabei einen durchaus wahrnehmbaren Einfluss auf die Kommunikation, insofern beispielsweise durch automatische Worterkennungssoftware oder die Möglichkeit, besonders gestaltete oder animierte Emoticons einzubauen, das Erscheinungsbild der SMS-Nachrichten mitbestimmt wird.

Der Begriff der Kommunikationsform wird von Dürscheid als Sammelkategorie für unterschiedliche "äußere Rahmen des kommunikativen Geschehens" (Dürscheid 2005: 5) verwendet. Kommunikationsformen werden "ausschließlich über textexterne Merkmale bestimmt" (Dürscheid 2003: 41). Diese Merkmale umfassen beispielsweise

- die Anzahl der KommunikationspartnerInnen (handelt es sich um eine Teilnehmerkonstellation, die als one-to-one, one-to-many oder many-to-many bezeichnet werden kann?),

\footnotetext{
${ }^{1}$ Dieser Medienbegriff hat nichts mit dem von Luhmann zu tun. Wenn Luhmann (1994: 23) vom "Medium Liebe" spricht, ist vom systemtheoretischen Medienbegriff die Rede, nicht vom technischen Begriff der Medien.
} 
- die Kommunikationsrichtung (dialogisch - monologisch),

- die verfügbaren Kommunikationskanäle (auditiv, visuell über Schrift oder visuell über multimodale Kommunikation) und

- die Geschwindigkeit des Nachrichtenaustauschs (asynchron, synchron oder quasisynchron).

Das SMS-Schreiben kann auf der Basis dieser Merkmale als Kommunikationsform gefasst werden, bei der typischerweise zwei Personen einander wechselseitig Nachrichten zuschicken können. Wichtig ist allerdings, dass die Kommunikationsform SMS auf Grund ihrer Mobilität und der damit einhergehenden Einbettung der SMS-SchreiberInnen in jeweils wechselnde Situationen sowie der technischen Integration des SMS-Dienstes in ein Mobiltelefon, das zugleich auch die Kommunikationsform des Telefongesprächs bereitstellt, einen schnellen Wechsel der Kommunikationsform ermöglicht. So kann beispielsweise in einer SMS ein Anruf angekündigt werden oder nach einem Anruf eine vergessene Information per SMS nachgereicht werden. Ebenso dienen SMS-Nachrichten häufig dazu, sich zu verabreden, also kurze Zeit später eine Face-to-Face-Kommunikationssituation herzustellen.

Kommunikationsformen stellen den Kommunikationsrahmen bereit, innerhalb dessen sich bestimmte Handlungsmuster in Form von kommunikativen Gattungen verfestigen können (vgl. Dürscheid 2005: 7). Der Begriff der kommunikativen Gattung geht dabei zurück auf die Arbeiten von Luckmann (1986, 1988), Günthner (1995, 2005, 2006) und Günthner/Knoblauch (1994, 1997). Kommunikative Gattungen werden dort als Strukturen aufgefasst, mit denen bestimmte kommunikative Probleme in einer Gesellschaft dadurch gelöst werden, dass sich mehr oder weniger feste Routinen und Orientierungsmuster herausbilden, an denen sich die Interagierenden orientieren. Diese Routinen erleichtern die Arbeit des Formulierens und sie erstrecken sich von der lexikalischen bis hin zur sequenziellen Ebene bzw. zu ganzen Handlungsmustern. Innerhalb der Kommunikationsform SMS können je nach situativer und funktionaler Einbettung zahlreiche unterschiedliche Gattungen entstehen: Verabredungs-SMS, Entschuldigungs-SMS, Plauder-SMS und, wie im vorliegenden Artikel relevant, Liebes-SMS. Das zentrale Problem, das bei Liebeskommunikation im Sinne des Gattungsansatzes gelöst werden muss, besteht nach Luhmann (1994: 211) darin, glaubhaft zu vermitteln, dass man den anderen aufrichtig liebt. In Liebes-SMS haben sich daher zahlreiche konventionalisierte Strukturen herausgebildet, die von Anredeformen (Schatzi) und Verabschiedungen (Bussi, Kuss) über phrasale und syntaktische Strukturen (ich vermiss dich so sehr) bis hin zu sequenziellen Mustern wie dem rituellen Aufkündigen der Beziehung (siehe Abschnitt 4.1) reichen können und deren Zweck genau darin besteht, Liebesbezeugungen als aufrichtig erscheinen zu lassen.

\section{Die Daten}

Die Datengrundlage bilden zwei SMS-Datensammlungen von jeweils deutschen und chinesischen SMS-Nachrichten, die im Rahmen des von Susanne Günthner geleiteten, vom DAAD und dem China Scholarship Council geförderten Projekts "Kommunikation in den Neuen Medien: Eine kontrastive Untersuchung von chinesischen und deutschen SMSBotschaften" entstanden sind.

Die chinesischen SMS wurden von Studierenden der International Studies University Xi'an erhoben. Die SchreiberInnen stammen hauptsächlich aus dem nordwestlichen Teil der Volksrepublik China (Gebiet um Xi'an), sind zwischen 20 und 30 Jahre alt und studieren. Daneben finden sich auch, in geringerer Anzahl, SchreiberInnen, die entweder bereits im Referendariat sind oder in einem Betrieb arbeiten sowie Eltern und Großeltern der Studierenden. Die SMS wurden jeweils von Hand in Excel-Tabellen übertragen. Insgesamt 
wurden so ca. 9000 SMS-Nachrichten erhoben. Metadaten liegen lediglich in Bezug auf das Alter, die Herkunft und den Berufsstand der SchreiberInnen vor (vgl. auch Hauptstock/König/Zhu 2010: 8-9 zu einer Beschreibung der Datensammlung).

Die deutschen SMS wurden zunächst in ähnlicher Weise erhoben, wobei sich die Sammlung in Form von Excel-Tabellen schnell als großes Hindernis bei der weiteren Arbeit herausstellte. Daher wurde von Marcel Fladrich im Jahr 2010 für die deutschen SMS eine Datenbank programmiert (http://cesi.uni-muenster.de/ SMSDB, Stand: 9. November 2012), die Recherchemöglichkeiten sowie ein umfangreiches Set an Metadaten bereitstellt. ${ }^{2}$ Die SMS wurden vor allem von Studierenden innerhalb von Seminaren an der Universität Münster erhoben. Entsprechend überwiegen auch hier SMS-Nachrichten von Studierenden. Eltern, Großeltern und befreundete Angestellte bilden eine kleinere Gruppe von Personen. Das Alter der SMS-SchreiberInnen, die meist aus dem Großraum Münster bzw. dem nördlichen Ruhrgebiet kommen, liegt ebenfalls vor allem im Bereich zwischen 20 und 30 Jahren. Inzwischen enthält die stetig weiter wachsende Datenbank 1627 SMS-Sequenzen mit einer Gesamtzahl von 6828 Einzel-SMS.

Die Metadaten umfassen Einträge zu Alter, Geschlecht, Beruf, Herkunft und Beziehung der Schreibenden zueinander und zuletzt zum Texteingabemodus des Mobiltelefons. Außerdem sind die SMS nach Kategorien geordnet, die sich auf den Kommunikationsanlass beziehen (Streit, Verabredung, Liebe etc.). Die SMS-Sequenzen werden in der Datenbank in Kolumnen geordnet dargestellt, wobei die linke Kolumne für SchreiberIn 1, die rechte für SchreiberIn 2 verwendet wird. Die Nachrichten werden fortlaufend durchnummeriert und enthalten Informationen über das Sendedatum und die Uhrzeit. Liegen diese Daten nicht vor, was im deutschen Korpus sehr selten vorkommt, häufig aber im chinesischen, ${ }^{3}$ wird statt Datum und Uhrzeit der Eintrag "ohne Timecode" verwendet.

Die Datenrecherche erwies sich auf Grund der unterschiedlichen Strukturen der chinesischen SMS-Sammlung und der deutschen SMS-Datenbank als schwierig. Während in den deutschen SMS über die Metadaten gezielt nach Liebeskommunikation gesucht werden konnte, mussten die chinesischen Daten von Hand durchsucht werden. Diese Probleme führten dazu, dass in dieser Untersuchung lediglich eine einzelfallbasierte, stichprobenartige Analyse vorgenommen werden kann, eine statistische Auswertung ist nicht möglich.

Sowohl die chinesische Datensammlung als auch die deutsche Datenbank haben das Problem, dass die Daten nicht konsequent anonymisiert wurden und auch das Einverständnis zur öffentlichen Bereitstellung in Form von Datenbanken nicht gegeben wurde. Daher sind beide Datenbanken nicht öffentlich zugänglich, was aus forschungsethischen Gründen zu bedauern ist.

Um dieses Problem zu beheben, wurde daher Ende 2011 eine öffentliche Version der SMSDatenbank, die für ForscherInnen zugänglich sein wird, von Dagmar Hüpper und Wolfgang Imo eingerichtet. Diese Datenbank weist die gleiche Architektur auf wie die nicht-öffentliche, die Besonderheit ist hier allerdings, dass die SMS-Nachrichten anonymisiert wurden und die Bereitschaft der SMS-SchreiberInnen, die Daten frei zur Verfügung zu stellen, eingeholt wurde. Die Datenbank enthält bislang 412 SMS-Sequenzen, die aus insgesamt 1884 einzelnen SMS-Nachrichten bestehen und steht seit der Mitte des Jahres 2012 über den "LinguistikServer Essen (LINSE)" (URL: http://www.linse.uni-due.de/index.html, Stand: 9. November 2012) zur allgemeinen Nutzung bereit.

\footnotetext{
2 Für die chinesische Seite scheiterte die Verwaltung der SMS in einer Datenbank statt einer einfachen Datensammlung in einer Excel-Tabelle bislang an infrastrukturellen und organisatorischen Problemen.

3 Aus Gründen der optisch kohärenten Präsentation der Daten wurden die chinesischen SMS-Nachrichten in das Darstellungsformat eingebunden, das für die deutschen SMS entwickelt wurde. Die originale Darstellung der chinesischen SMS-Sequenzen ist durch das Excel-Format sehr unübersichtlich.
} 
Es ist zu hoffen, dass Folgeprojekte es ermöglichen, eine parallele öffentliche Datenbank auch für die chinesischen SMS-Nachrichten bereitzustellen, so dass umfangreichere kontrastive Untersuchungen durchgeführt werden können. Für die SMS-Kommunikation in der Schweiz liegt beispielsweise eine solche kontrastive Datenbank mit dem multilingualen Projekt sms4science (http://www.sms4science.ch, Stand: 9. November 2012) bereits vor, das von Dürscheid/Stark (2011) beschrieben wird. Aufbauend auf dem Projekt ist ein von Stähli/Dürscheid/Béguelin (2011) herausgegebener Sammelband zum Thema "SMSKommunikation in der Schweiz: Sprach- und Varietätengebrauch" erschienen. Die momentane Datenlage, auf die die vorliegende Untersuchung aufbaut, erlaubt dagegen (vor allem auf Grund der sehr schwer recherchierbaren chinesischen SMS-Daten) bislang nur explorative Untersuchungen.

\section{Liebeskommunikation: eine sequenzielle Analyse von Liebes-SMS}

Selbstverständlich gibt es eine große Zahl unterschiedlicher Routinen und Muster, mit denen sowohl die chinesischen als auch die deutschen SMS-SchreiberInnen sich gegenseitig ihrer Liebe versichern. Die Kommunikationsform SMS als eine "social technology par excellence" (Thurlow/Poff i. E.: 10) ist dabei prädestiniert für diese Aufgabe. Von besonderer Bedeutung zeigt sich in den untersuchten Daten die Aktivierung einer spielerischen Modalität, mit der die Interaktionspartner sich gegenseitig ihre Vertrautheit signalisieren und Nähe herstellen. Während diese Merkmale für deutsche und chinesische Liebes-SMS gleichermaßen konstatiert werden können, so zeigen sich doch auch in den Korpora bestimmte Unterschiede in der Art und Weise, wie genau spielerisch miteinander umgegangen wird. Besonders auffällig war in dem chinesischen Korpus die scherzhafte Drohung mit der Aufkündigung der Beziehung, die in den deutschen SMS-Sequenzen nicht vorkam.

Im Folgenden wird bei den chinesischen Liebes-SMS daher auf dieses besondere kommunikative Muster der scherzhaften Beziehungsaufkündigung fokussiert, während bei den deutschen Liebes-SMS der Untersuchungsrahmen weiter gefasst werden muss. Eine Aufkündigung der Beziehung kam in dem deutschen SMS-Korpus zwar auch einmal vor, allerdings nur zwischen eng befreundeten SchreiberInnen, nicht zwischen Liebespaaren. Diese SMS-Sequenz wird ausnahmsweise - obwohl ihr keine Liebesbeziehung im engeren Sinn zu Grunde liegt - ebenfalls in die Analyse der deutschen SMS aufgenommen, um Gemeinsamkeiten und Unterschiede einer solchen Androhung der Aufkündigung der Beziehung im Deutschen und Chinesischen zu illustrieren.

\subsection{Liebeskommunikation per SMS - chinesisch}

Wie bereits erwähnt, war ein Muster in den chinesischen SMS besonders auffällig, in dem der Schreiber/die Schreiberin seiner Partnerin/ihrem Partner damit drohte, die Beziehung zu beenden. Diese Beziehungsaufkündigung ist manchmal in eine bereits laufende Sequenz eingebettet, manchmal eröffnet sie aber auch eine Sequenz als erster Zug. ${ }^{4}$ In beiden Fällen kann dabei festgestellt werden, dass die Drohung der Beziehungsaufkündigung durch die extreme Knappheit und Situationsentbundenheit der SMS-Kommunikation sehr unvermittelt und überraschend wirkt. Dies wird durch das Fehlen von Gestik, Mimik und Prosodie noch verstärkt. Non- und paraverbale Mittel dienen in Face-to-Face-Interaktionen als Kontextualisierungshinweise (ausführlich dazu Gumperz 1982) unter anderem dazu, einen Wechsel in eine andere Interaktionsmodalität oder Missverständnisse bzw. aufkommenden Dissens anzuzeigen. Dadurch, dass diese Kontextualisierungshinweise entweder ganz fehlen oder als graphostilistische Elemente (Ausrufezeichen, Emoticons) weniger projizierende als eher ad hoc kommentierende Wirkung haben, da sie den Äußerungen nachgestellt werden,

\footnotetext{
${ }^{4}$ Eine Übersicht über die sequenzielle und interaktionale Organisation von SMS-Interaktionen findet sich in Günthner (2011).
} 
entsteht der Eindruck eines abruptem Modalitätswechsels. Die folgende SMS-Sequenz illustriert einen solchen abrupten Übergang, bei dem allerdings zumindest über Onomatopoetica und eine folgende begründende Handlung eine gewisse Kontextualisierungsleistung gegeben ist. Die SMS-SchreiberInnen sind eine Studentin (20 Jahre alt, Beiträge in der linken Spalte) und ihr Freund, ein Student (21 Jahre alt, Beiträge in der rechten Spalte):

\section{Sequenz 15}

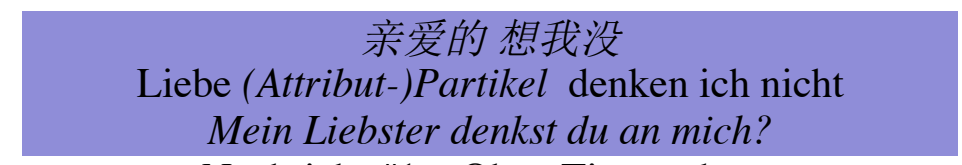

Nachricht \#1 - Ohne Timecode
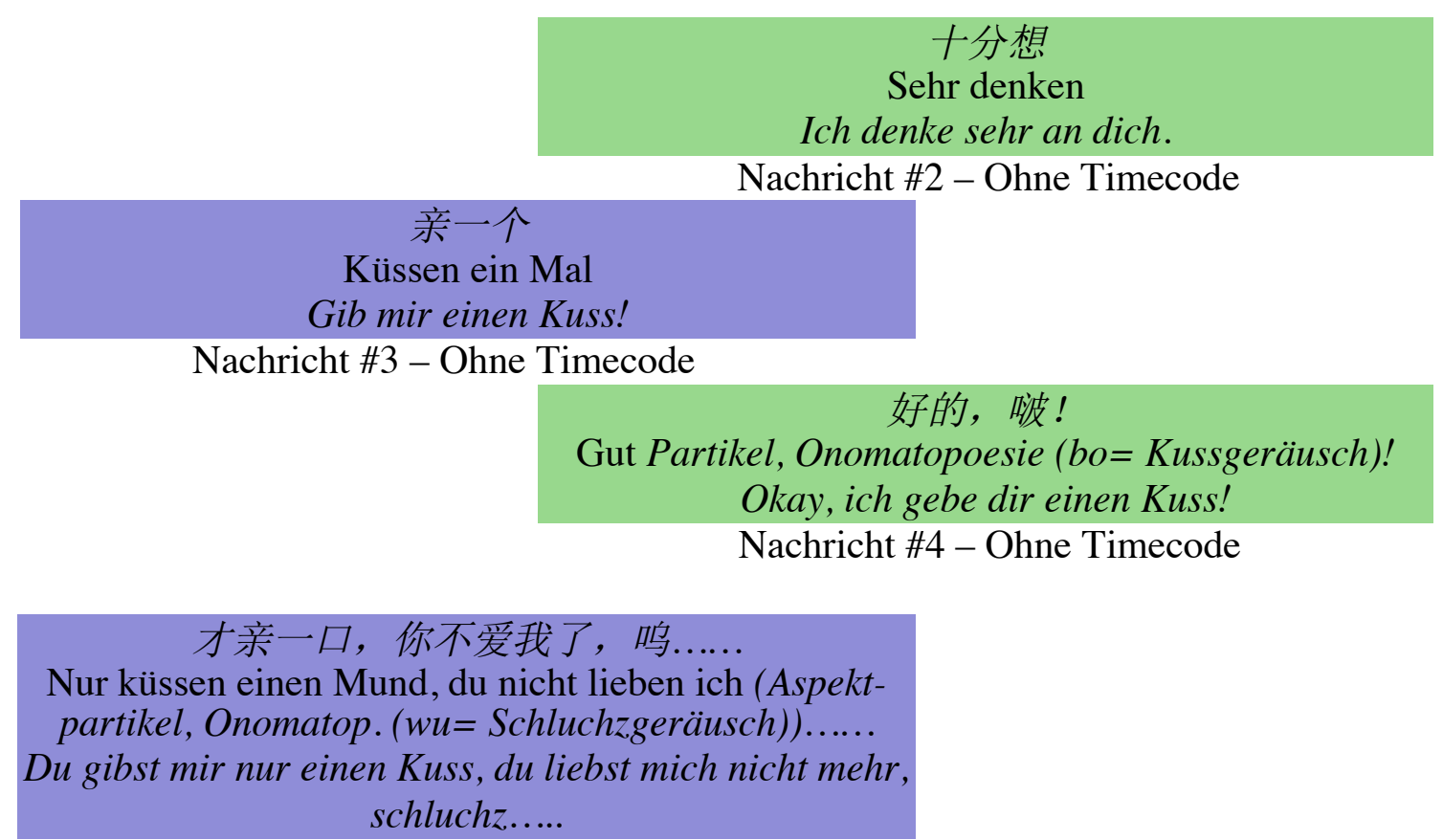

Nachricht \#5 - Ohne Timecode

别呀! 亲爱的, 我很爱你!
Nicht Partikel! Lieben (Attribut-)Partikel, ich sehr
lieben du!
Nicht doch! Geliebte, ich liebe dich sehr!

\section{你得了吧！你是不是又喜欢上别人了,} 你别骗我了, 我都知道了。

Du es genügen Partikel! Du sein nicht sein wieder mögen anderen Menschen (Aspekt-)Partikel, du nicht anlügen ich Partikel, ich alles wissen (Aspekt-)Partikel. Das genügt! Hast du dich in jemand anderes verliebt?!

Lüg mich nicht an, ich weiß es schon.

Nachricht \#7 - Ohne Timecode

没有呀, 宝贝，我发誓:我只喜欢你，你过来，你让
我亲多少口都可以!
Nein Partikel, mein Schatz, ich schwören: ich nur
lieben du, du herkommen, du lassen ich küssen
wieviele Münder alles gehen!
Nicht doch, Schatz, ich schwöre dir: ich liebe nur dich,

\footnotetext{
${ }^{5}$ Ich danke Saskia Kriese für die Bereitstellung und Übersetzung der chinesischen SMS-Sequenzen.
} 


komm zu mir, ich kann dir so viele Küsse geben, wie du
willst!
Nachricht $\# 8$ - Ohne Timecode

我们分手吧!
Wir trennen Partikel!
Lass uns doch trennen!
Nachricht \#9 - Ohne Timecode
不要呀, 亲爱的宝贝。
Nicht müssen Partikel,
Ich sehr lieben sehr lieb
Leb das nicht, geliebter
sehr, werde dich me
Nachricht \#
肉, 我就不和你分手了
Gut, ich vergeben du (Aspekt-)Partikel, heute
好, 我原谅你了,
einem Fleischspieß, ich dann nicht mit du trennen
Partikel Na gut, ich vergebe dir, lade mich heute
Nachmittag zu Youpomian mit einem extra Fleischspieß
ein, dann trenne ich mich auch nicht mehr von dir.

Nachricht \#11 - Ohne Timecode

好的，我爱死你了，宝贝。我请你吃什么都可以。

Gut Partikel, ich lieben tot du (Aspekt-)Partikel, mein

Schatz. Ich einladen du essen was alles können.

Gut, ich liebe dich sehr, Schatz. Wenn ich dich zum

Essen einlade, dann kannst du alles haben.

我手机没电了

Nachricht \#12 - Ohne Timecode

Ich Handy nicht Strom (Aspekt-)Partikel

Mein Akku hat keinen Strom mehr.

Nachricht \#13 - Ohne Timecode

那 Bye!
Dann Bye!
Dann Bye!

Nachricht \#14 - Ohne Timecode

Der Einstieg in die Sequenz ist nicht ungewöhnlich: In den untersuchten Daten beginnen Liebes-SMS-Sequenzen häufig nach der Anredefloskel (wie im vorliegenden Fall durch 亲爱的 'Mein Liebster' in Nachricht 1) mit einer Thematisierung der Abwesenheit des anderen, entweder durch eine Frage (Denkst $d u$ an mich? Vermisst $d u$ mich?) oder eine Aussage (Ich denke die ganze Zeit an dich! Ich vermisse dich!). Die ersten beiden SMS-Züge entsprechen somit hochgradig routinierten Mustern der Liebeskommunikation. Die Antworten des Partners orientieren sich dabei an den Konventionen für präferierte zweite Paarteile, insofern die Antworten jeweils gleichlaufend geäußert werden. Wie Auer/Uhmann (1982) für das Deutsche und Pomerantz (1984) für das Englische zeigen, muss bei präferierten gleichlaufenden zweiten Bewertungen eine Verstärkung bzw. "Eskalation" (Auer/Uhmann 1982: 8) der ursprünglichen Proposition durchgeführt werden. Ohne eine Verstärkung der 
Proposition in der Reaktion würde die Aussage eher als Ankündigung einer NichtÜbereinstimmung gewertet werden. Dieses Prinzip lässt sich auch in der Antwort des Partners gut beobachten: Er "eskaliert" das Verb denken in sehr denken und führt damit die für eine Übereinstimmung notwendige Verstärkung durch.

Auch die Aufforderung Gib mir einen Kuss ist in deutschen und chinesischen Liebes-SMS nicht ungewöhnlich und führt meist zu verbalen (Kuss) oder graphostilistischen "Küssen", wie in folgendem Beispiel aus einer deutschen Liebes-SMS (Student, 25 Jahre alt rechte Spalte und Studentin, 22 Jahre alt, linke Spalte), in der wechselseitig erst ein, dann zwei, dann drei und schließlich wieder ein Kuss ausgetauscht werden:

\section{Sequenz 2}

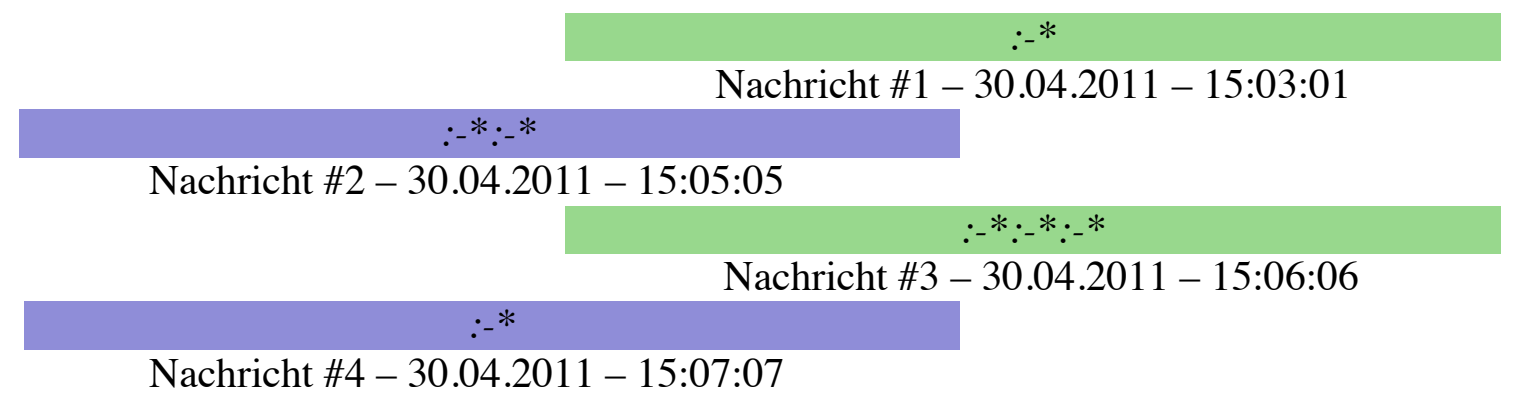

Auch hier kann man das Grundprinzip der Eskalation gut beobachten: Es genügt nicht, mit einer gleichlaufenden Form zu reagieren, ein Kuss muss mindestens durch zwei Küsse überboten werden. ${ }^{6}$

In der vorliegenden chinesischen SMS-Sequenz 1 allerdings reagiert der Partner auf die Einforderung eines Kusses in Nachricht 3 nicht mit einer Eskalation, also vielen Küssen, sondern mit einer neutralen gleichlaufenden Reaktion: 好的, 皮! 'Okay, ich gebe dir einen Kuss!' (Nachricht 4). Dies ist der Auslöser für den Wechsel der Interaktionsmodalität: Während die einleitende Frage nach dem Fortbestand der Liebe und der Austausch von Küssen ernst gemeint waren, reagiert nun die Partnerin in einer spielerischen Modalität auf die ausbleibende Eskalation und kommentiert die Tatsache, dass sie "nur einen Kuss" erhalten hat, mit der Schlussfolgerung 你不爱我了, 呜...... 'du liebst mich nicht mehr, schluchz.....' (Nachricht 5). Sie zeigt mit dieser Reaktion deutlich ihre Orientierung an der "Eskalationsregel" von Liebesbeweisen an. ${ }^{7}$ Dass es sich um eine scherzhafte Modalität handelt, wird zum einen über die abrupte Reaktion und zum anderen das onomatopoetische schluchz sowie die interaktionsperpetuierenden Punkte ("....") deutlich.

Der Partner reagiert daraufhin damit, dass er die Unterstellung seiner Partnerin, er liebe sie nicht mehr, zurückweist (别呀! 'Nicht doch!', Nachricht 6) und seine Liebe erneut beteuert (亲爱的, 我很爱你 'Geliebte, ich liebe dich sehr!', Nachricht 6). Diese Bekräftigung wird von der Partnerin nicht akzeptiert, sie baut ihre Anschuldigungen weiter aus, indem sie unterstellt, dass sich ihr Partner in eine andere Frau verliebt hat und dass sie davon sogar Kenntnis habe. Auf diese Verschärfung der Anklage reagiert der Partner, indem er den "einen" Kuss, der der Auslöser für den gespielten Streit war, in so viele Küsse [...] wie du

\footnotetext{
6 Ähnlich auch Auer (1988: 14), der feststellt, dass "eine Äußerung wie ich liebe dich keine Beschreibung eines affektiven Zustands ist, sondern eine Haltung dem Gesprächspartner gegenüber ausdrückt", die als solche eine festgelegte Reaktion erfordert. Entspricht diese Reaktion nicht den Erwartungen, führt dies zu deutlichen Problemen: "Tatsächlich ist das Fehlen einer 'angemessenen' Erwiderung auf eine Liebeserklärung (also eine Erwiderung wie soso, oder das Fehlen einer Erwiderung) konversationell dramatisch und führt zu Reparaturmaßnahmen [...]." (Auer 1988: 15)

7 Auer/Uhmann (1982: 8) zeigen, dass bei einem Austausch von Bewertungen die zweite Bewertung als eine versteckte gegenläufige Bewertung interpretiert werden kann, wenn sie neutral statt eskalierend geäußert wird. SprecherInnen können also eine neutrale (von Auer/Uhmann 1982: 8 auch "niveaugleich" genannte) zweite Reaktion dafür verwenden, ihre Nicht-Übereinstimmung auf sehr höfliche Weise und sehr implizit anzuzeigen.
} 
willst (Nachricht 8) eskaliert - jedoch zu spät und ohne Erfolg. Der gespielte Streit gipfelt in dem Vorschlag der Partnerin, sich zu trennen (我们分手吧! 'Lass uns doch trennen!', Nachricht 9), der abrupt und ohne auf die Liebesbeteuerungen des Partners einzugehen in dem Folgebeitrag geliefert wird. Diesem bleibt nichts weiter, als seine Liebesbeteuerungen weiter zu eskalieren (爱你一生一世'werde dich mein ganzes Leben lang lieben'; Nachricht 10), und erst diese Beteuerung hat einen - ebenso abrupten - Erfolg. Die Partnerin verzeiht ihm (好, 我原谅你了'Na gut, ich vergebe dir', Nachricht 11), nicht ohne jedoch die Situation auszunutzen, um neben den Liebesbeteuerungen auch noch eine Einladung zum Essen (mit "extra Fleischspieß") einzufordern.

Die gesamte Strategie erinnert an ein Muster, das Pomerantz (1980) mit dem Begriff des "fishing", des "Fischzuges" nach Informationen bezeichnet hat. Pomerantz bezeichnet damit die Technik, mit Hilfe von scheinbar unschuldig wirkenden Aussagen das Gegenüber dazu zu bringen, private Informationen preiszugeben. Beispiele für solche von Jefferson (1980: 189; Übersetzung W.I.) beschriebenen Strategien sind Ich habe dich schon vorher versucht anzurufen, aber es war niemand da oder Du warst ganz schön lang in Zimmer 252 heute Nachmittag. Mit solchen scheinbar harmlosen Feststellungen wird das Gegenüber in einen gewissen Zugzwang gebracht, die Wissenslücken des Sprechers zu füllen und die fehlenden Informationen nachzureichen.

Die Tatsache, dass diese besonders stark auf wechselseitige Orientierung basierende Strategie des "fishing" auch in den SMS-Sequenzen vorkommt, kann als weiterer Beleg für Günthners (2011, i. V.; vgl. auch Spagnolli/Gamberini 2007) These angesehen werden, dass SMSSequenzen als interaktionale Strukturen - und daher mit den Bordmitteln der Gesprächsanalyse und Interaktionalen Linguistik - analysiert werden sollten:

Auch wenn es sich bei der SMS-Interaktion um eine schriftliche, wechselseitig mittelbare Kommunikationsform handelt, die sowohl zeitlich als auch räumlich distant abläuft, handelt es sich keineswegs um monologische Texte. Vielmehr zeichnen sich SMS-Beiträge durch eine sequenzielle Folge von Dialogzügen aus, welche am zeitlichen Davor ausgerichtet sind, konditionelle Relevanzen für die Folgezüge setzen und sich mit dem jeweils spezifischen Rezipientendesign am Gegenüber orientieren. (Günthner i. V.: 18)

Man kann sagen, dass die Partnerin in der eben untersuchten SMS-Sequenz 1 nachdrücklich nach Informationen "fischt", speziell nach Informationen darüber, ob die Liebe ihres Partners noch so stark ist, wie sie sein sollte (bzw. ob er sich die Mühe gibt, ihr seine Liebe adäquat zu vermitteln). Die ausbleibende Eskalation, also die Tatsache, dass nur ein Kuss und nicht mehrere Küsse geliefert werden, wird von der Partnerin als Indiz für nachlassende Liebe gewertet. Sie verstärkt daher den Druck und fordert mittels des Druckmittels der Trennung adäquate Liebesbeweise sowie Wiedergutmachungsleistungen ein.

Interessant ist dabei auch die Beendigung der SMS-Sequenz: Nachdem die Einladung zum Essen bekräftigt ist, beendet die Partnerin sehr abrupt mit der Information 我手机没电了 'Mein Akku hat keinen Strom mehr' (Nachricht 13) den Austausch, ohne dass von ihrer Seite - aber auch nicht von der ihres Partners - noch abschließende Liebesbeweise notwendig werden. Dieser Ausstieg unterstreicht den zweckorientierten Anschein, den die gesamte Sequenz erweckt: Die Ziele sind erreicht, Liebesbeweise wurden eingeholt und das Mittagessen ist gesichert, die Interaktion kann daher beendet werden.

Die abrupten Übergänge erscheinen aus deutscher Sicht "ungewöhnlich" und könnten als Indiz für eine entsprechend routinierte Form der Liebes-Interaktion aufgefasst werden. $\mathrm{Zu}$ Grunde liegt dabei das Wissen, dass es sich bei Liebe - mit Luhmann (1994) gesprochen um eine "unwahrscheinliche Anforderung" handelt, die in der Realität tatsächlich oft in kommunikativen Störungen oder gar Zusammenbrüchen endet. In der hier vorgestellten SMS- 
Sequenz wird somit auf ein allseits bekanntes Muster des Zusammenbruchs der Kommunikation in Liebesbeziehungen verwiesen, das spielerisch nachgebaut wird und gerade dadurch paradoxerweise stabilisierend wirken kann, also die bestehende Liebe fortführt.

Wie routiniert die Trennungsdrohung zu sein scheint, kann man an folgendem minimalen Austausch sehen. In diesem Fall ist es nicht die Partnerin (eine Studentin, 20 Jahre, rechte Spalte), die mit der Trennung "droht", sondern ihr Partner (ein Ingenieur, 21 Jahre alt, linke Spalte):

\section{Sequenz 3}

对不起！我们分手吧！别问我原因，没有为什么！
别哭 你还是以前的你！对不起
Entschuldigung! Wir trennen (Aufforderungs-)Partikel!
Nicht fragen ich Grund, nicht haben warum! Nicht
weinen Du noch sein früher (Attribut-)Partikel du!
Entschuldigung
Entschuldige! Wir trennen uns/Lass uns doch trennen!
Frage nicht nach dem Grund, es gibt kein "warum"!
Weine nicht Du bist noch dieselbe wie früher!
Entschuldige

Nachricht \#1 - Ohne Timecode

呵呵, 恩
Onomatopoesie, Partikel
Hehe, okay.

Nachricht \#2 - Ohne Timecode

Die Trennungsaufforderung kommt unerwartet, es wird sogar explizit darauf verwiesen, dass es keinen Grund für die Trennung gibt (别问我原因, 没有为什么! 'Frag nicht nach dem Grund, es gibt kein 'warum"; Nachricht 1). Gleichzeitig weist allerdings die in chinesischen SMS weit verbreitete, vage "Involviertheit" und "Nähe"8 anzeigende Tilde (别哭 'Weine nicht $\sim$ ', Nachricht 1) darauf hin, dass der Partner weiterhin emotionale Verbundenheit mit der Partnerin signalisiert. Dies ist jedoch der einzige Kontextualisierungshinweis, der die ansonsten sehr abrupt wirkende Trennungsankündigung als möglicherweise doch nicht ganz ernst gemeint indizieren könnte (wenn auch nur sehr vage und implizit). Dennoch hat seine Partnerin offensichtlich überhaupt keine Probleme damit, die scherzhafte Modalität zu erkennen, und hält es auch nicht für nötig, mit weiteren Zügen auf das "Spiel" einzugehen und es auszubauen: Ihr Lachen zeigt an, dass sie die Drohung als nicht ernst gemeint auffasst. Mit 恩 'okay' bestätigt sie in der Antwort in Nachricht 2 lapidar das Trennungsangebot. Ein Grund dafür, dass hier kein Ausbau der Trennungsthematik mit Rückeroberungen oder Wiedergutmachungen erfolgt, könnte darin bestehen, dass dieses Muster so weit verfestigt ist, dass es nur noch angedeutet, aber nicht mehr vollständig ausgespielt werden muss. Ein weiterer Grund könnte zudem aber auch sein, dass hier die Rollen verkehrt sind: Die typische Konzeptionalisierung von Liebe, so Luhmann (1994: 77), sieht vor, dass der Mann die Frau erobert, gleichzeitig sich aber dem Willen der Frau unterwirft - beide Merkmale sind in der zuvor analysierten Sequenz 1 gegeben, nicht aber in Sequenz 3. Es bleibt daher hier bei der bloßen Andeutung eines als bekannt vorausgesetzten Musters, das in diesem Kontext nur noch der Erheiterung dient.

\footnotetext{
${ }^{8} \mathrm{Zu}$ den Funktionen der Tilde in chinesischen SMS-Nachrichten vgl. Hauptstock/König/Zhu (2010: 22) sowie Günthner/Kriese (i. V.).
} 


\subsection{Liebeskommunikation per SMS - deutsch}

Wie bereits erwähnt, finden sich scherzhaft gemeinte Trennungsdrohungen nicht in deutschen Liebes-SMS. Der einzige Fall, in dem mit der Aufkündigung einer Beziehung gedroht wurde, fand zwischen zwei lediglich befreundeten SMS-SchreiberInnen statt (ein Angestellter, 28 Jahre alt, linke Spalte und eine Studentin, 24 Jahre, rechte Spalte). Der Freund wollte die Freundin einladen, mit ihm gemeinsam auf ein Festival zu fahren. Diese sagte ihm einige Stunden vor dem Zeitpunkt ab, an dem er die folgenden SMS schrieb:

\section{Sequenz 4}

leb wohl. danke.
Nachricht \#1 - 29.04.2011 - 01:15:05
vergess einfach alles von mir.
Nachricht \#2 - 29.04.2011 - 01:23:15
ich werde es vermeiden mich nochmal auf dich zu
freuen. bless.
Nachricht \#3 - 29.04.2011 - 01:23:35
ich lass dortmund und alle erwartungen fallen.
Nachricht \#4 - 29.04.2011 - 01:59:01
ich fahr da hin. Und nur da hin. aber ich nehm es dir
nicht übel. Deine Entscheidung ob wir uns sehen.

Nachricht \#5 - 29.04.2011 - 02:26:34

tommi, ich bin jetzt nach hause gegangen und lade mein handy auf. weshalb schreibst du mir solche dinge? du bist willkommen. was lässt dich zweifeln? hab stress mit anna wegen des wochenendes. weiß nicht, was ich tun soll. was rätst du mir als freund? Nachricht \#6 - 29.04.2011 - 02:46:05

weiß zwar von gestern nicht mehr viel, aber trotzdem ein großes sorry...

Nachricht \#7 - 29.04.2011 - 11:02:13

bitte schick mir deine Bankverbindung. überweise es dir gleich wenn ich die hab. vorfreude ist ne gefährliche sache :-*

Nachricht \#8 - 29.04.2011 - 12.00 .00

Zwei wichtige Unterschiede zu Sequenz 1 fallen hier auf: zum einen die Tatsache, dass der Freund seine fünf Nachrichten zwischen 1:15 und 2:25 in der Nacht abgeschickt hat. Seine Antwort auf die verwunderte Rückfrage der Freundin klärt die Bedeutung der Uhrzeit: wei $\beta$ zwar von gestern nicht mehr viel, aber trotzdem ein großes sorry... (Nachricht 7). Er impliziert damit, dass er diese Nachrichten in betrunkenem Zustand geschrieben hat und dass diese aus diesem Grund in ihrer Geltung aufgehoben werden müssen.

Der zweite Unterschied betrifft die Reaktion der Freundin, die nicht mit Entschuldigungen oder gar Wiedergutmachungsangeboten reagiert, sondern mit Verwunderung und einem Vorwurf (weshalb schreibst du mir solche dinge?, Nachricht 6). ${ }^{9}$ Die Intentionen der SprecherInnen erweisen sich somit im Verlauf von Sequenz 4 als grundlegend andere als in Sequenz 1, auch wenn die Möglichkeit besteht, dass der Freund mit seinen SMS tatsächlich ein schlechtes Gewissen erzeugen wollte und somit auf einen "Fischzug" nach Entschuldigungen und einer möglichen Wiedergutmachung aus war, die beispielsweise darin

\footnotetext{
${ }^{9}$ Zum Muster von Vorwürfen in Frageformaten siehe Günthner (2000: 85-94).
} 
hätte bestehen können, dass die Freundin ihre Absage zurücknimmt, um nun doch mit ihm zu dem Festival zu fahren.

Grundsätzlich kann gesagt werden, dass wann immer in dem untersuchten Korpus in deutschen Liebes-SMS scherzhafte Modalitäten oder Frotzeleien vorkommen, diese eher in Wortspielen oder Kalauern verbleiben. Eine mitlaufende Agenda wie die eines "Fischzugs" nach Liebesbeweisen wird nicht so sehr in den Vordergrund gestellt wie in den chinesischen Beispielen (wobei sie auf der anderen Seite aber auch nie völlig ausgeblendet wird). In der folgenden SMS-Sequenz sind eine Studentin (22 Jahre, linke Spalte) und ein Student (25 Jahre, rechte Spalte) beteiligt. Als Hintergrundinformation zum Verständnis der SMSBeiträge muss angemerkt werden, dass die Partnerin sich vor kurzem neue Schuhe bestellt hat, die sie mit ihrem Partner am Tag zuvor anprobiert hat. Zudem hat sich ihr Partner gerade seine lockigen Haare kurz schneiden lassen.

\section{Sequenz 5}

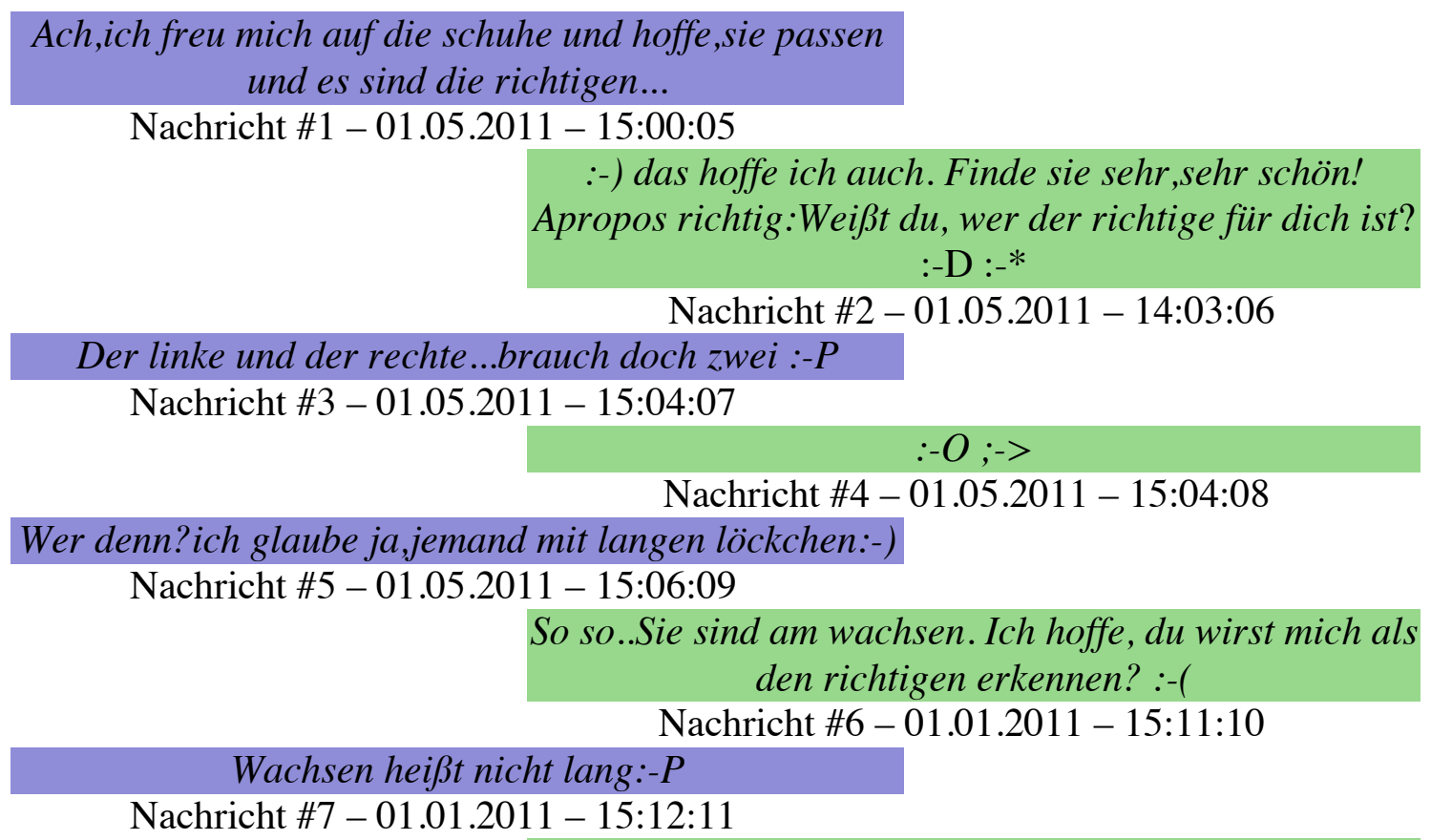

$T z z z$

Nachricht \#8 - 01.01.2011 - 15:13:12

Ein "Fischzug" nach Liebesbeweisen wird von dem Partner in seiner Antwort-SMS auf die erste SMS seiner Partnerin, in der sie ihre Freude über die erwarteten Schuhe ausdrückt, mit der Frage Weißt du wer der richtige für dich ist? (Nachricht 1) gestartet, zudem wird die SMS mit dem Emoticon für einen Kuss $\left(:^{*}\right)$ beendet, der als weiterer "Köder" bei diesem "Fischzug" - Auer (1988: 29) spricht von dem "exhortativen Charakter", den solche Liebeserklärungen haben - gewertet werden kann. Gleichzeitig stuft der Partner seine Äußerungen aber durch zwei Strategien zurück: Die erste besteht im Einsatz der Floskel Apropos richtig (Nachricht 2), mit der ein Abschweifen vom Thema und damit indirekt Beiläufigkeit signalisiert wird. Die zweite Strategie betrifft das Emoticon aus der gleichen Nachricht, das lautes Lachen ausdrückt $(:-D)$ und so den Themenwechsel und das Wortspiel als Scherz kontextualisiert. In dem Wortspiel greift der Partner die Äußerung "die richtigen" seiner Partnerin auf, mit der sie auf ihre Schuhe Bezug nahm, um die Phrase in "der richtige" umzuwandeln, mit der er sich selbst meint.

Der Grad der Verpflichtung, mit Liebesbeweisen (bzw. mit Eskalationen vorheriger Liebesbeweise) $\mathrm{zu}$ reagieren, wird durch diese Beiläufigkeit und scherzhafte Modalität anzeigenden Strategien reduziert. Die Partnerin orientiert sich auch in der Tat an dem 
Wortspiel und interpretiert "der richtige" absichtlich falsch als der richtige Schuh, wobei sie darauf hinweist, dass sie zwei Schuhe benötigt (Nachricht 3). Das Emoticon, das eine herausgestreckte Zunge symbolisiert (:-P), zeigt dabei an, dass sie das Wortspiel sehr wohl verstanden hat, es aber für ihre Zwecke als Grundlage für ein weiteres Wortspiel ausnutzt. Ihr Partner honoriert diesen Konter mit zwei Emoticons, die Erstaunen und eine Mischung aus Zwinkern und Lachen darstellen (:- $O$;->, Nachricht 4).

Interessant ist allerdings, dass der "Fischzug" des Partners letzten Endes dennoch erfolgreich ist, ohne dass er erneut initiiert werden muss, ein Beleg für Auers (1988: 17) These, dass Liebeserklärungen "den Anderen unter Zugzwang" stellen: In Nachricht 5 wechselt nämlich die Partnerin von sich aus von der "Kalauer-Lesart" in die von ihrem Partner intendierte Lesart der Frage nach dem richtigen Partner. Sie greift seine Frage wieder auf und beantwortet sie mit einer Frotzelei, die auf die kurzen Haare ihres Partners hinweist (ich glaube ja,jemand mit langen löckchen) und die durch den lachenden Smiley (:-)) als scherzhaft kontextualisiert wird. Wieder bietet ihr Partner zwei Ebenen an, auf die reagiert werden kann: Zum einen verweist er auf seine bereits wieder wachsenden Haare, zum anderen "fischt" er aber auch in Nachricht 6 weiter nach Bestätigung in der Liebe: Ich hoffe, $d u$ wirst mich als den richtigen erkennen? :-(. Seine Partnerin entscheidet sich auch jetzt dafür, nicht auf diesen "Köder" zu reagieren und kommentiert - mit herausgestreckter Zunge :-P (Nachricht 7) - seine Haare als noch nicht lang genug, lässt also offen, ob er wirklich der Richtige ist bzw. ob sie ihn als Richtigen erkennen kann. Ihr Partner gibt schließlich auf (Tzzz, Nachricht 8). Dem SMS-Austausch folgt im Übrigen kurze Zeit später ein Telefonanruf (ein klassischer Fall von "Mixing Media" nach Wyss 2011: 102).

Auch die letzte hier diskutierte SMS-Sequenz weist Züge des "Kalauerns", des Spiels mit Wörtern auf, das die Aktivitäten der Liebesbezeugungen überlagert. Die Partnerin (linke Spalte) ist 22 Jahre alt und Studentin, der Partner (rechte Spalte) 26 Jahre alt und Student:

\section{Sequenz 6}

Will ein süppchen mit entenförmigen nudeln ...gibt keine
entenförmigen nudeln-hab ich gerade so
überlegt...braucht man aber!sind bestimmt witzig...

Nachricht \#1 - 05.05.2011 - 22:31:05

Du bist mir ein huhn!:-)

Nachricht \#2 - 05.05.2011 - 22:32:05

Ein suppenhuhn?;-)

Nachricht \#3 - 05.05.2011 - 22:45:05

Und was für eins ;-)

Nachricht \#4 - 05.05.2011 - 22:46:05

\section{Besser als ne pute!}

Nachricht \#5 - 05.05.2011 - 22:47:05

Du könntest gar keine dumme Pute sein!

Nachricht \#6 - 05.05.2011 - 22:49:06

Danke,das schmeichelt mir doch sehr!pokpok;-):):-*

Nachricht \#7 - 05.05.2011 - 22:55:07

Anders als in dem vorigen Beispiel geht hier allerdings keiner der beiden Partner explizit auf einen "Fischzug", um Liebesbezeugungen einzufordern. Die Eröffnungs-SMS in diesem Austausch hat den Charakter einer Mitteilung im "Plaudermodus", die darauf verweisen könnte, dass die Schreiberin plant, eine Suppe zu kochen. Luhmann (1994: 200) liefert eine Erklärung für solche scheinbar belanglosen Kommunikate: "Liebende können unermüdlich miteinander reden, weil alles Erlebte mitteilenswert ist und kommunikative Resonanz findet." 
Der Partner reagiert auf die Mitteilung mit einem Kalauer, er greift den Begriff der "entenförmigen" Nudeln auf und typisiert mit "Du bist mir ein huhn!" seine Partnerin, ${ }^{10}$ wobei er diese Typisierung durch das lachende Emoticon :-) (Nachricht 2) als Scherz kontextualisiert. Diese steigt in den spielerischen Modus ein und verbindet die Themen "Suppe" und "Huhn" in der Frage Ein suppenhuhn? (Nachricht 3). Der zwinkernde Smiley ;-) markiert das Wortspiel als nicht ganz ernst zu nehmen. Ihr Partner steigt darauf ein und bestätigt diese Selbstbezeichnung (Und was für eins ;-), Nachricht 4), woraufhin seine Partnerin - weiter im Wortfeld des Geflügels verbleibend - mit Besser als ne pute! (Nachricht 5) auf einen Vogel verweist, der, anders als "Huhn" immer als Beleidigung verwendet wird. Mit dem Wort (bzw. der typischen Kollokation "dumme Pute") werden eingebildete und dumme Menschen bezeichnet. Ihr Partner beeilt sich in Nachricht 6 auch dementsprechend, diese Zuschreibung emphatisch abzulehnen: Du könntest gar keine dumme Pute sein!. Dadurch zeigt er an, dass trotz der in diesem Austausch vorherrschenden scherzhaften Modalität immer die Gefahr besteht, die "falsche" Antwort zu geben - wie es dem Partner in Sequenz 1 passierte. Auf der anderen Seite macht er auch deutlich, dass er auf perfekt auf der Klaviatur der Liebesbezeugungen spielen kann: Die Reaktion seiner Partnerin kann als voller Erfolg gewertet werden, vom Dank für das Kompliment bis zum abschließenden Kuss (:-*) wurde trotz des zunächst auf Wortspielen beruhenden Austauschs der Bogen zur Liebeskommunikation geschlagen und die Fortdauer der Liebesbeziehung gesichert.

\section{Gemeinsamkeiten und Unterschiede deutscher und chinesischer Liebes-SMS}

Was die in dieser Untersuchung diskutierten SMS-Sequenzen auszeichnet, ist etwas, das Wyss (2011: 98) generell für Liebeskommunikation beschrieben hat, nämlich eine "starke Ästhetisierung" durch besondere stilistische Mittel und Redefiguren. Dies liegt daran, dass

Liebe selbst kein Gefühl [ist], sondern ein Kommunikationscode, nach dessen Regeln man Gefühle ausdrücken, bilden, simulieren, anderen unterstellen, leugnen und sich mit all dem auf die Konsequenzen einstellen kann, die es hat, wenn entsprechende Kommunikation realisiert wird. (Luhmann 1994: 23)

Insofern ist es nicht verwunderlich, wenn sich in deutschen und chinesischen Liebes-SMS ganz ähnliche Strategien der Ästhetisierung und der Routinierung von sprachlichen Strategien, vor allem von Strategien der Codierung von Emotionen (vgl. Derks/Fischer/Bos $2008 \mathrm{zu}$ einem Überblick über die Rolle von Emotionen in der computervermittelten Kommunikation) finden lassen. Dieses Grundmerkmal teilen die hier untersuchten chinesischen und deutschen Liebes-SMS gleichermaßen. Die Unterschiede bestehen lediglich darin, dass im Chinesischen eine routinierte - wenn nicht gar ritualisierte - Drohung mit der Aufgabe der Beziehung als ein Mittel bereitsteht. Es handelt sich dabei um ein Muster, das in der deutschen Liebeskommunikation - zumindest auf der Basis der dieser Arbeit zu Grunde liegenden Daten - nicht verbreitet zu sein scheint.

Gemeinsam haben die hier untersuchten deutschen und chinesischen Liebes-SMS auch den Einsatz orthographischer Mittel ${ }^{11}$ zur Kodierung von Emotionen. Mit diesen Mitteln können Emotionen - allerdings sehr kontextspezifisch und nur über den konkreten Kontext

\footnotetext{
10 Als "Huhn" kann man Menschen (meist Frauen) bezeichnen, die unorganisiert und hektisch sind, wild gestikulieren und lachen ("gackern"). Meist wird die Bezeichnung sogar eher wohlwollend als Charakterisierung eingesetzt, Äußerungen wie "du bist ein verrücktes Huhn" können sogar bewundernd gemeint sein.

11 Eine ältere, aber ausführliche Studie zu orthographischen und graphostilistischen Besonderheiten der SMSKommunikation findet sich in Schlobinski et al. (2001). Im Vergleich zur SMS-Kommunikation werden in der gesprochenen Sprache dagegen vor allem prosodische Mittel zur Kontextualisierung von Liebesemotionen eingesetzt: "Im love talk kontextualisieren die Liebenden ihre Rollen fortwährend durch bestimmte Merkmale ihres Miteinander-Sprechens. Dazu gehören (schwer zu fassende) phonetische Merkmale wie 'verträumte Stimme', falsetto, Flüstern, bestimmte Arten von Lächeln und Lachen, kinder- und paarsprachliche Ausdrucksformen und natürlich non-verbale Verhaltensformen." (Auer 1988: 21)
} 
interpretierbar - markiert werden, wie z. B. in Nachricht 2 aus der SMS-Sequenz 5: Finde sie sehr,sehr schön!. Das Ausrufezeichen hebt die Aussage hervor und unterstreicht so die positive Bewertung. Ganz anders in Nachricht 7 aus Sequenz 1, wo das Ausrufezeichen bei Das genügt! die (gespielte) Entrüstung wiedergibt und bei Hast du dich in jemand anderes verliebt?! (Nachricht 7 in Sequenz 1), wo die Kombination aus Fragezeichen und Ausrufezeichen den Zwischenstatus zwischen unsicherem und sicherem Wissen markiert. In der Folgeäußerung Lüg mich nicht an, ich weiß es schon. (Nachricht 7 in Sequenz 1) wiederum indiziert der Punkt Finalität und sicheres Wissen. Eine besondere Rolle fällt den Bereichen der Graphostilistik und der onomatopoetischen Ausdrücke zu. Beide haben gemeinsam, dass mit ihnen die Multimodalität prototypischer Face-to-Face-Interaktion zumindest teilweise in die zeitlich zerdehnten und allein auf die Schriftkommunikation beschränkten SMS-Sequenzen zurückgeholt werden kann. Auch hier gilt, dass diese multimodalen Faktoren (primär Prosodie, sekundär Mimik und, seltener, Gestik oder ganze Handlungen) überwiegend der Codierung von Emotionen dienen: So wird durch den "Inflektiv" (Schlobinski 2001) schluchz (Sequenz 1) Trauer angezeigt, durch pokpok (Nachricht 7 in Sequenz 6) der scherzhafte Austausch über Huhn vs. Pute gerahmt und mit Hilfe von :- $O$;-> (Nachricht 5 in Sequenz 5) Überraschung über den Vorgängerbeitrag und zugleich das Verständnis dieses Beitrags als Scherz signalisiert. Als wichtigster Bestandteil der Liebeskommunikation werden Küsse als symbolische Handlungen wiedergegeben, manchmal onomatopoetisch, wie in Nachricht 4 in Sequenz 1 (皮), manchmal als Emoticon und dann kombiniert mit weiteren Emoticons (Lachen und Kuss: :-D :-*, Nachricht 2 in Sequenz 5) oder als ganze Serie von Emoticon-Küssen (:-*:-:-*, Nachricht 3 in Sequenz 2).

Auch bezüglich der Aufgaben der Liebeskommunikation und der rhetorischen Strategien, die die Liebenden einsetzen, gibt es Parallelen zwischen den hier untersuchten deutschen und chinesischen SMS-Sequenzen. Eine zentrale Aufgabe von Liebes-Kommunikation besteht darin, Liebesbezeugungen zu übermitteln oder einzufordern und sich die fortdauernde Liebe bestätigen zu lassen. Diese Aufgabe kann den Hauptteil einer Kommunikation ausmachen (wie z. B. in den Sequenzen 1 und 5) oder aber "mitlaufen" und an passender Stelle zum Vorschein treten (wie in Sequenz 6).

Eine Strategie, besondere Nähe und Vertrautheit - und somit, implizit als Inferenz mitlaufend, Liebe - zu signalisieren, besteht in der Anwendung spielerischer Modalitäten und der Inszenierung ganzer Sequenzen, die nur aus Wortspielen oder Kalauern bestehen. Vertrautheit markierende Scherze oder Frotzeleien wie Du bist mir ein huhn! (Nachricht 2 aus Sequenz 6), die Antwort, dass nur jemand mit langen löckchen der richtige Partner sein kann, obwohl dieser gerade die Haare kurz geschnitten hat (Nachricht 5 aus Sequenz 5) oder auch die gespielte Enttäuschung, nur einen Kuss erhalten zu haben Du gibst mir nur einen Kuss, $d u$ liebst mich nicht mehr ${ }^{12}$ (Nachricht 5 aus Sequenz 1) sorgen dafür, dass immer mehr "Insider"-Wissen entsteht und spezialisierte Kommunikationsrituale aufgebaut werden können.

Ein zentrales Problem besteht jedoch grundsätzlich in der Frage, inwieweit es möglich ist, dem Partner zu vermitteln, dass man ehrlich liebt und aufrichtig seine Liebe gesteht. ${ }^{13}$ Dies erklärt, weshalb routinierte Formen der Einforderung und Bestätigung von Liebe entstehen

${ }^{12}$ Gerade bei dieser Äußerung ist gut zu beobachten, dass sich Frotzeleien typischerweise "zwischen Vorwurf und Spiel" (Günthner 2000: 155) bewegen und somit durchaus das Potential haben, aus dem spielerischen Rahmen in einen ernsthaften Rahmen hinüberzuwechseln. Frotzeln ist also gerade in der Liebeskommunikation (und generell bei intimen Beziehungen) auch immer eine prekäre Handlung, die fehlschlagen kann.

13 "Soweit Liebeserklärungen als Ausdruck von Emotionen gelten können, impliziert dieses Vorgehen auch, dass deren Darstellung in der Interaktion alles andere als 'natürlich' ist, sondern in hohem Maß bestimmten vorgegebenen Mustern folgt." (Auer 1988: 12) 
konnten: Das kommunikative Problem, das Liebes-SMS zu lösen haben, besteht darin, aufrichtig Liebe zu zeigen bzw. umgekehrt zu überprüfen, ob der Partner noch aufrichtig liebt. In den SMS-Sequenzen fanden sich darauf bezogene Routinefloskeln wie Gib mir einen Kuss!, Geliebte, ich liebe dich sehr!, ich liebe nur dich (alle Sequenz 1) oder Weißt du, wer der richtige für dich ist? (Sequenz 5). Dass diese Floskeln genau aus dem Grund, dass es sich um Floskeln handelt, bereits ihre Funktion verloren haben, geeignete Lösungen für das Problem der Kommunikation von Aufrichtigkeit darzustellen, merkt man an der Tatsache, dass jede Liebeseinforderung und Liebesbeteuerung eine jeweils eskalierte zweite Beteuerung nach sich ziehen muss. Macht ein Partner in einer Liebeskommunikation den Fehler und "eskaliert" nicht, so kann dies als lediglich höfliche, aber unaufrichtige Kommunikation gewertet werden. Dies führt letztendlich in eine "Rhetorik des Exzesses" (Luhmann 1994: 71), in der beispielsweise mit Hilfe von Gradpartikeln (Ich liebe dich wirklich sehr, Nachricht 10 aus Sequenz 1), Extremformulierungen ${ }^{14}$ (werde dich mein ganzes Leben lang lieben, Nachricht 10 aus Sequenz 1) oder "expressiven Markern" wie ich schwöre (vgl. Bahlo 2010) die notwendige zusätzliche kommunikative Arbeit geleistet wird, die schließlich die Aufrichtigkeit als wahrscheinlich erscheinen lässt: Schatz, ich schwöre dir: ich liebe nur dich (Nachricht 8 aus Sequenz 1). ${ }^{15}$ Diese Rhetorik des Exzesses hängt dabei auch eng mit der Konzeptualisierung von Liebe als Kampf, Eroberung und Unterwerfung zusammen (vgl. Abschnitt 4.1). Die Rituale der Eroberung und Unterwerfung können beliebig oft nachgespielt werden und liefern durch ihre stetige Re-Inszenierung die nötige Bestätigung, dass die Liebe fortdauert. Sie können - wie im Fall der chinesischen SMS-Sequenzen 1 und 3 - letztendlich auch in routinierte Trennungsrituale münden. Durch ihre interaktionalen, d.h. eng an den Vorgängeräußerungen ausgerichteten, lokal extrem adaptiven Strukturen "tragen [SMSInteraktionen; W. I.] zur Konstruktion und Bestätigung sozialer Beziehungen bei", indem sie "Reziprozität" herstellen und "sich mit ihrem spezifischen Rezipientendesign an den InteraktionspartnerInnen" (Günthner 2011: 33) ausrichten. Diese Flexibilität und Anpassungsfähigkeit machen sie zu einer so sehr geeigneten Kommunikationsform der Liebeskommunikation.

\section{$6 \quad$ Ausblick}

Die vorliegende Untersuchung kann nur als ein erster Schritt in Richtung einer sprach- und kulturvergleichenden Studie von Liebes-Interaktionen per SMS angesehen werden. Gerade hinsichtlich der sequenziellen Ablaufmuster, verfestigten Routinen und Konzeptualisierungen von Liebe und "angemessener" Liebeskommunikation lässt sich viel über sprachliche und kulturelle Unterschiede herausfinden. Damit solche Studien möglich werden, ist es allerdings nötig, dass Korpora aufgebaut werden, die einen solchen Vergleich zulassen. Die SMSKorpora, die in Münster und Xi'an erhoben wurden, erfüllen die Bedingung der Vergleichbarkeit insofern, als sie beide informelle SMS-Kommunikation primär zwischen Studierenden enthalten. Was als Desiderat jedoch für die Zukunft erstrebenswert wäre, ist die Erstellung systematischer, durchgängig übersetzter, öffentlich zugänglicher und mit umfangreichen Metadaten versehener Korpora, d.h. Korpora, in denen z. B. deutsche und chinesische Liebes-SMS, Verabredungs-SMS, Plauder-SMS etc. jeweils parallel in deutscher und chinesischer Sprache vorliegen. Erst dann ist es möglich, in dem Bereich sprach- und

\footnotetext{
14 Siehe Pomerantz (1986) zu "extreme case formulations".

15 Bezüglich der Verwendung von "ich schwöre" - allerdings in einem jugendsprachlichen, nicht liebesbezogenen Kontext - schreibt Bahlo (2010: 118f.): "Die rekategorisierte ich-schwöre-Konstruktion erfüllt dabei verschiedene metapragmatische Funktionen, die teils retraktiv, teils projizierend den Diskurs organisieren, in dem sie bekräftigend und bestätigend Stellung zu Äußerungen nehmen und Gemütszustände, die sich auf vorangegangene oder Folgesyntagmen beziehen, kommunizieren kann. Durch die verschiedenen Intonationsmöglichkeiten von ich-schwöre können Emotionen bereits im Vor-Vorfeld als Signal für den Hörer angekündigt werden."
} 
kulturvergleichender Erforschung computervermittelter Kommunikation per SMS (vgl. zu einem kurzen Überblick über die bestehende Forschung in diesem Bereich Thurlow/Poff i. E.: 3-4) zahlreiche bislang bestehende Forschungslücken zu schließen, die in diesem Beitrag nur in Ansätzen thematisiert werden konnten.

\section{Literatur}

Auer, Peter (1988): "Liebeserklärungen. Oder: Über die Möglichkeiten, einen unmöglichen Handlungstyp zu realisieren". Sprache und Literatur 61: 11-31.

Auer, Peter/Uhmann, Susanne (1982): "Aspekte der konversationellen Bewertungen". Deutsche Sprache 10: 1-32.

Bahlo, Nils Uwe (2010): "uallah und / oder ich schwöre. Jugendsprachliche expressive Marker auf dem Prüfstand". Gesprächsforschung - Online-Zeitschrift zur verbalen Interaktion 11: 101-122. www.gespraechsforschung-ozs.de, Stand 13. November 2012.

Derks, Daantje/Fischer, Agneta H./Arjan, E. R. Bos (2008): "The role of emotion in computer-mediated communication: A review". Computers in Human Behaviour 24: 766785.

Dürscheid, Christa (2003): "Medienkommunikation im Kontinuum von Mündlichkeit und Schriftlichkeit. Theoretische und empirische Probleme". Zeitschrift für Angewandte Linguistik 38: 37-56.

Dürscheid, Christa (2005): "Medien, Kommunikationsformen, kommunikative Gattungen". Linguistik online 22/1: 3-16. http://www.linguistik-online.de/22_05/duerscheid.pdf, Stand 13. November 2012.

Dürscheid, Christa/Stark, Elisabeth (2011): "sms4science: An international corpus-based texting project and the specific challenges for multilingual Switzerland". In: Thurlow, Crispin/Mroczek, Kristine (eds.) (2011): Digital Discourse. Language in the New Media. Oxford, Oxford University Press: 299-320.

Günthner, Susanne (1995): "Gattungen in der sozialen Praxis". Deutsche Sprache 3: 193-218.

Günthner, Susanne (2000): Vorwurfsaktivitäten in der Alltagsinteraktion. Tübingen: Niemeyer.

Günthner, Susanne (2005): "Kommunikative Gattungen in interkulturellen Kommunikationssituationen". In: Bismark, Heike et al. (eds.) (2005): Usbekisch-deutsche Studien. Münster, Lit-Verlag: 43-62.

Günthner, Susanne (2006): "Von Konstruktionen zu kommunikativen Gattungen: Die Relevanz sedimentierter Muster für die Ausführung kommunikativer Aufgaben". Deutsche Sprache 34: 173-190.

Günthner, Susanne (2011): "Zur Dialogizität von SMS-Nachrichten - eine interaktionale Perspektive auf die SMS-Kommunikation". Networx 60: 1-37. http://www.mediensprache.net/networx/networx-60.pdf, Stand 13. November 2012.

Günthner, Susanne (i. V.): "'Lupf meinen Slumpf' - die interaktive Organisation von SMSDialogen".

Günthner, Susanne/Knoblauch, Hubert (1994): "'Forms are the food of faith'. Gattungen als Muster kommunikativen Handelns". Kölner Zeitschrift für Soziologie und Sozialpsychologie 4: 693-723.

Günthner, Susanne/Knoblauch Hubert (1997): "Gattungsanalyse". In: Hitzler, Ronald/Honer, Anne (eds.) (1997): Qualitative Methoden und Forschungsrichtungen in den Sozialwissenschaften. Opladen, Leska \& Budrich: 281-308.

Günthner, Susanne/Kriese, Saskia (i. V.): "Dialogizität in der chinesischen und deutschen SMS-Kommunikation - eine kontrastive Studie".

Gumperz, John J. (1982): Discourse Strategies. Cambridge, Cambridge University Press.

Hauptstock, Amelie/König, Katharina/Zhu, Quiang (2010): "Kontrastive Analyse chinesischer und deutscher SMS-Kommunikation - ein interaktionaler und gattungstheoretischer 
Ansatz". Networx 58: 1-44. http://www.mediensprache.net/networx/networx-58.pdf, Stand 13. November 2012.

Holly, Werner (1997): "Zur Rolle von Sprache in Medien. Semiotische und kommunikationsstrukturelle Grundlagen". Muttersprache 1: 64-75.

Luhmann, Niklas (1994): Liebe als Passion. Frankfurt/Main: Suhrkamp.

Luckmann, Thomas (1986): "Grundformen der gesellschaftlichen Vermittlung des Wissens: Kommunikative Gattungen". Kölner Zeitschrift für Soziologie und Sozialpsychologie 27: 191-211.

Luckmann, Thomas (1988): "Kommunikative Gattungen im kommunikativen 'Haushalt' einer Gesellschaft". In: Smolka-Koerdt, Gisela/Spangenberg, Peter M./Tillmann-Bartylla, Dagmar (eds.) (1988): Der Ursprung von Literatur. München, Fink: 179-288.

Pomerantz, Anita M. (1980): "Telling My Side: 'Limited Access' as a 'Fishing Device'". Sociological Inquiry 50: 186-198.

Pomerantz, Anita M. (1984): "Agreeing and disagreeing with assessments". In: Atkinson, J. Maxwell/Heritage, John (eds.) (1984): Structures of Social Action. Cambridge, Cambridge University Press: 57-101.

Pomerantz, Anita M. (1986): "Extreme case formulations: A way of legitimizing claims". Human Studies 9 (2-3): 219-229.

Schlobinski, Peter (2001): "*knuddel - zurueckknuddel - dich ganzdollknuddel*. Inflektive und Inflektivkonstruktionen im Deutschen". Zeitschrift für germanistische Linguistik 29: 192-218.

Schlobinski, Peter et al. (2001): "Simsen. Eine Pilotstudie zu sprachlichen und kommunikativen Aspekten in der SMS-Kommunikation". NETWORX 22: 1-32. Verfügbar unter: http://www.mediensprache.net/networx/networx-22.pdf.

Spagnolli, Anna/Gamberini, Luciano (2007): "Interacting via SMS. Practices of social closeness and reciprocation". In: British Journal of Social Psychology 46: 343-364.

Stähli, Adrian/Dürscheid, Christa/Béguelin Marie-José (eds.) (2011): SMS-Kommunikation in der Schweiz: Sprach- und Varietätengebrauch. (= Linguistik online 48 (Themenheft)).

Thurlow, Crispin/Poff, Michele (i.E.): "The Language of Text Messaging". In: Herring, Susan C./Stein, Dieter/Virtanen, Tuija (eds.) (i. E): Handbook of the Pragmatics of CMC. Berlin: de Gruyter.

Wyss, Eva L. (2011): "Brautbriefe, Liebeskorrespondenzen und Online-Flirts. Schriftliche Liebeskommunikation vom 19. Jahrhundert bis in die Internet-Ära". In: Luginbühl, Martin/Perrin, Daniel (eds.) (2011): Muster und Variation: Medienlinguistische Perspektiven auf Textproduktion und Text. Bern, Lang: 81-124. 